\title{
TWO CONSEQUENCES OF DAVIES'S HARDY INEQUALITY
}

\author{
RUPERT L. FRANK AND SIMON LARSON
}

In memory of M. Z. Solomyak, on the occasion of his 90th birthday

\section{INTRODUCTION}

In this short note we would like to show that one can use Davies's Hardy inequality to rederive well-known results of Lieb [7] and Rozenblum [9]. Throughout the following we fix an open set $\Omega \subset \mathbb{R}^{d}$ and define, for $\omega \in \mathbb{S}^{d-1}$,

$$
\delta(x):=\left(\left|\mathbb{S}^{d-1}\right|^{-1} \int_{\mathbb{S}^{d-1}} d_{\omega}(x)^{-2} d \omega\right)^{-1 / 2} \quad \text { where } \quad d_{\omega}(x):=\inf \{|t|: x+t \omega \notin \Omega\}
$$

(with the convention that $\inf \emptyset=0$ ). Then Davies's Hardy inequality [1] states that

$$
\int_{\Omega}|\nabla u|^{2} d x \geq \frac{1}{4} \int_{\Omega} \delta^{-2}|u|^{2} d x \quad \text { for all } u \in H_{0}^{1}(\Omega) .
$$

The following simple lemma is key to our argument.

Lemma 1. For any $x \in \Omega$ and any $\rho>0$,

$$
\left|\Omega \cap B_{\rho}(x)\right| \geq\left(1-\rho^{2} \delta(x)^{-2}\right)\left|B_{\rho}(x)\right| .
$$

Proof. We have

$$
\left|\Omega \cap B_{\rho}(x)\right|=\int_{\mathbb{S}^{d-1}} \int_{0}^{\rho} \mathbb{1}_{\Omega}(x+t \omega) t^{d-1} d t d \omega
$$

and clearly, for any $\omega \in \mathbb{S}^{d-1}$ with $d_{\omega}(x)>\rho$, we have $x+t \omega \in \Omega$ for all $t \in(0, \rho)$. Thus,

$$
\left|\Omega \cap B_{\rho}(x)\right| \geq\left|\left\{\omega \in \mathbb{S}^{d-1}: d_{\omega}(x)>\rho\right\}\right| d^{-1} \rho^{d} .
$$

On the other hand, clearly,

$$
\rho^{-2}\left|\left\{\omega \in \mathbb{S}^{d-1}: d_{\omega}(x) \leq \rho\right\}\right| \leq \int_{\mathbb{S}^{d-1}} d_{\omega}(x)^{-2} d \omega=\left|\mathbb{S}^{d-1}\right| \delta(x)^{-2}
$$

or, equivalently,

$$
\left|\left\{\omega \in \mathbb{S}^{d-1}: d_{\omega}(x)>\rho\right\}\right| \geq\left(1-\rho^{2} \delta(x)^{-2}\right)\left|\mathbb{S}^{d-1}\right|
$$

Inserting this bound into (2) implies the lemma.

(C) 2020 by the authors. This paper may be reproduced, in its entirety, for non-commercial purposes.

U.S. National Science Foundation grants DMS-1363432 and DMS-1954995 (R.L.F.) and Knut and Alice Wallenberg Foundation grant KAW 2018.0281 (S.L.) are acknowledged. 


\section{A theorem of Lieb}

Let $-\Delta_{\Omega}^{D}$ be the Dirichlet Laplacian in $L^{2}(\Omega)$ and

$$
\lambda_{\Omega}:=\inf \operatorname{spec}\left(-\Delta_{\Omega}^{D}\right)=\inf \left\{\int_{\Omega}|\nabla u|^{2} d x: u \in H_{0}^{1}(\Omega), \int_{\Omega}|u|^{2} d x=1\right\} .
$$

It is well-known that if $\Omega$ is mean-convex, then $\lambda_{\Omega}$ is bounded from below by a constant times the inverse square of the radius of the largest ball contained in $\Omega$ and that this is not true for general open $\Omega$. It is a theorem of Lieb [7] that this remains true for general open $\Omega$, provided 'the largest ball contained in $\Omega$ ' is replaced by 'a ball that intersects $\Omega$ significantly'. Here we give a simple alternative proof of this result using (1) (albeit with a slightly worse constant).

Theorem 2. Let $\Omega \subset \mathbb{R}^{d}$ be open. Then for any $\rho>0$,

$$
\lambda_{\Omega} \geq \frac{1}{4 \rho^{2}}\left(1-\sup _{x \in \Omega} \frac{\left|\Omega \cap B_{\rho}(x)\right|}{\left|B_{\rho}(x)\right|}\right) .
$$

Clearly, this theorem implies for all $0<\theta<1$,

$$
\lambda_{\Omega} \geq \frac{1-\theta}{4 \rho_{\theta}^{2}}, \quad \text { where } \quad \rho_{\theta}:=\inf \left\{\rho>0: \sup _{x \in \Omega} \frac{\left|\Omega \cap B_{\rho}(x)\right|}{\left|B_{\rho}(x)\right|} \leq \theta\right\} .
$$

Proof. Inserting (11) into (3), we obtain

$$
\lambda_{\Omega} \geq \frac{1}{4} \inf \left\{\int_{\Omega} \delta^{-2}|u|^{2} d x: u \in H_{0}^{1}(\Omega), \int_{\Omega}|u|^{2} d x=1\right\} \geq \frac{1}{4} \inf _{\Omega} \delta^{-2} .
$$

Inserting the lower bound on $\delta^{-2}$ from Lemma 1 we obtain the theorem.

Remarks. (1) The theorem remains valid for the principal eigenvalue of the $p$ Laplacian with $1<p<\infty$. This follows from the validity of the analogue of (1) for $1<p<\infty$. Lieb's proof works in the case $p=1$ as well.

(2) If $\lambda$ is an eigenvalue of $-\Delta_{\Omega}$, then there is an $x \in \Omega$ such that for all $\rho>0$, $\lambda \geq\left(4 \rho^{2}\right)^{-1}\left(1-\left|\Omega \cap B_{\rho}(x)\right| /\left|B_{\rho}(x)\right|\right)$. This follows from the same method of proof, by noting that in this case the inequality $\lambda \geq(1 / 4) \int_{\Omega} \delta^{-2}\left|u_{0}\right|^{2} d x$ for a normalized eigenfunction $u_{0}$ implies that there is an $x \in \Omega$ with $\lambda \geq 1 /\left(4 \delta(x)^{2}\right)$.

(3) Lieb's result was improved upon in [8] in the sense that the overlap between $\Omega$ and $B_{\rho}(x)$ is quantified in terms of capacity instead of measure. It would be interesting to investigate whether there is a strengthening of (1) that implies this result.

\section{A theorem of Rozenblum}

We denote by $N_{\leq}\left(\lambda,-\Delta_{\Omega}^{D}\right)$ the total spectral multiplicity of $-\Delta_{\Omega}^{D}$ in the interval $[0, \lambda]$. It is well-known [9] that for $\Omega$ of finite measure, one has Weyl asymptotics $N_{\leq}\left(\lambda,-\Delta_{\Omega}^{D}\right) \sim(2 \pi)^{-d} \omega_{d}|\Omega| \lambda^{d / 2}$ as $\lambda \rightarrow \infty$, as well as a universal bound $N_{\leq}\left(\lambda,-\Delta_{\Omega}^{D}\right) \leq C_{d}|\Omega| \lambda^{d / 2}$ for all $\lambda>0$. A theorem of Rozenblum [9] implies, in particular, that sets $\Omega$ that satisfy the reverse inequality $N\left(\lambda,-\Delta_{\Omega}^{D}\right) \geq \varepsilon|\Omega| \lambda^{d / 2}$ for some $\lambda>0$ have a substantial 'well-structured' component at spatial scale $\lambda^{-1 / 2}$. 
Theorem 3. For any $\theta \in(0,1]$ there are constants $c_{1}(\theta), c_{2}(\theta, d)>0$ with the following property. For any open set $\Omega \subset \mathbb{R}^{d}$ and any $\lambda>0$ there are disjoint balls $B^{(1)}, \ldots, B^{(M)} \subset \mathbb{R}^{d}$ of radius $c_{1} \lambda^{-1 / 2}$ such that

$$
\left|\Omega \cap B^{(m)}\right| \geq(1-\theta)\left|B^{(m)}\right| \quad \text { for all } m=1, \ldots, M
$$

and

$$
M \geq c_{2} N_{\leq}\left(\lambda,-\Delta_{\Omega}^{D}\right) .
$$

Note that choosing $\lambda=\lambda_{\Omega}$ we obtain again Theorem 2, up to constants.

Proof. We begin by giving the proof in dimension $d \geq 3$, where we have

$$
N_{\leq}\left(\lambda,-\Delta_{\Omega}^{D}\right) \leq L_{d} \int_{\Omega}\left(\lambda-\frac{1}{4 \delta(x)^{2}}\right)_{+}^{\frac{d}{2}} d x .
$$

This appears in [5], but a weaker version with $1 / 4$ replaced by a smaller constant follows easily by (21) and the CLR inequality (see [3] for references).

Let $E:=\left\{x \in \Omega: \delta(x) \geq(4 \lambda)^{-1 / 2}\right\}$. Then, by Lemma 1,

$$
\left|\Omega \cap B_{\rho}(x)\right| \geq\left(1-4 \rho^{2} \lambda\right)\left|B_{\rho}(x)\right| \quad \text { for all } x \in E \text { and all } \rho>0 .
$$

For $\rho=(\theta /(4 \lambda))^{1 / 2}$ the claimed density condition is satisfied for each such ball.

Let $B_{\rho}\left(x_{m}\right)$ be a maximal disjoint subcollection of $B_{\rho}(x), x \in E$. Then $E \subset$ $\bigcup_{m} B_{2 \rho}\left(x_{m}\right)$ (since for any $x \in E$ there is an $x_{m}$ such that $B_{\rho}(x)$ intersects $B_{\rho}\left(x_{m}\right)$, so $\left|x-x_{m}\right|<2 \rho$, so $\left.x \in B_{2 \rho}\left(x_{m}\right)\right)$. In case there are infinitely many $x_{m}$ we are done. If there are finitely many $x_{m}$, say $M$, then

$$
\begin{aligned}
\int_{\Omega}\left(\lambda-\frac{1}{4 \delta(x)^{2}}\right)_{+}^{\frac{d}{2}} d x & =\int_{E}\left(\lambda-\frac{1}{4 \delta(x)^{2}}\right)^{\frac{d}{2}} d x \leq \lambda^{\frac{d}{2}}|E| \leq \lambda^{\frac{d}{2}} \sum_{m}\left|B_{2 \rho}\left(x_{m}\right)\right| \\
& =\omega_{d} 2^{d} \lambda^{\frac{d}{2}} \rho^{d} M=\omega_{d} \theta^{\frac{d}{2}} M .
\end{aligned}
$$

Together with (4) this gives the claimed lower bound on $M$ for $d \geq 3$.

For $d=2$ (the case $d=1$ is easy) we bound $N_{\leq}\left(\lambda,-\Delta_{\Omega}^{D}\right) \leq \lambda^{-\gamma} \operatorname{Tr}\left(-\Delta_{\Omega}^{D}-2 \lambda\right)_{-}^{\gamma}$ for any $\gamma>0$ and use the fact [5] that

$$
\operatorname{Tr}\left(-\Delta_{\Omega}^{D}-\mu\right)_{-}^{\gamma} \leq L_{\gamma, 2} \int_{\Omega}\left(\mu-\frac{1}{4 \delta(x)^{2}}\right)_{+}^{\gamma+1} d x .
$$

The claimed bound now follows similarly as before.

Remarks. (1) In Rozenblum's formulation, the balls are required to be centered on $\left(c \lambda^{-1 / 2}\right) \mathbb{Z}^{d}$. This can also be achieved by a minor modification of our proof.

(2) In fact, Rozenblum proves a stronger theorem where the overlap between $\Omega$ and $B_{\rho}(x)$ is quantified in terms of capacity instead of measure. It would be interesting to investigate whether there is a corresponding strengthening of (4).

(3) A related result for Schrödinger operators was proved in [2].

(4) Theorem 3 might be useful in the problem of maximizing $\operatorname{Tr}\left(-\Delta_{\Omega}-\lambda\right)_{-}^{\gamma}$ among sets $\Omega$ of given measure; see [6, 4] for partial results for $\gamma \geq 1$. 


\section{REFERENCES}

[1] E. B. Davies, Some norm bounds and quadratic form inequalities for Schrödinger operators. II. J. Operator Theory 12 (1984), no. 1, 177-196.

[2] C. L. Fefferman, The uncertainty principle. Bull. Amer. Math. Soc. (N.S.) 9 (1983), no. 2, 129206.

[3] R. L. Frank, The Lieb-Thirring inequalities: Recent results and open problems. Preprint (2020), arXiv:2007.09326.

[4] R. L. Frank, S. Larson, Two-term spectral asymptotics for the Dirichlet Laplacian in a Lipschitz domain. J. Reine Angew. Math. 766 (2020), 195-228.

[5] R. L. Frank, M. Loss, Hardy-Sobolev-Maz'ya inequalities for arbitrary domains. J. Math. Pures Appl. (9) 97 (2012), no. 1, 39-54.

[6] S. Larson, Asymptotic shape optimization for Riesz means of the Dirichlet Laplacian over convex domains. J. Spectr. Theory 9 (2019), no. 3, 857-895.

[7] E. H. Lieb, On the lowest eigenvalue of the Laplacian for the intersection of two domains. Invent. Math. 74 (1983), no. 3, 441-448.

[8] V. Maz'ya, M. Shubin, Can one see the fundamental frequency of a drum? Lett. Math. Phys. 74 (2005), no. 2, 135-151.

[9] G. V. Rozenbljum, The eigenvalues of the first boundary value problem in unbounded domains. Mat. Sb. (N.S.) 89 (131) (1972), no. 2, 234-247. English translation: Math. USSR Sb. 18 (1972), $235-248$.

(R. L. Frank) Mathematisches Institut, Ludwig-Maximilans Universität München, Theresienstr. 39, 80333 München, Germany, and Department of Mathematics, California Institute of Technology, PAsadena, CA 91125, USA

Email address: r.frank@lmu.de, rlfrank@caltech.edu

(S. Larson) Department of Mathematics, California Institute of Technology, Pasadena, CA 91125, USA

Email address: larson@caltech.edu 